\title{
Transformación digital de la administración pública: ¿Qué competencias necesitan los empleados públicos?
}

\section{Digital transformation of public administration: Which competences do civil workers need?}

\author{
Fernando Trujillo Sáez \\ Universidad de Granada (España) \\ ORCID: https://orcid.org/0000-0002-8972-3218 \\ ftsaez@ugr.es
}

\section{NOTA BIOGRÁFICA}

Doctor por la Universidad de Granada. I Premio Universidad de Granada-Caja Rural de Granada a la Comunicación e Innovación en Medios Digitales (Edición 2016). Codirige el Máster Propio en Promoción de la Salud y Salud Comunitaria de la EASP y la Universidad de Granada. Coordina el grupo de investigación HUM-840 «Conocimiento Abierto para la Acción Social».

David Álvarez Jiménez Universidad de Granada (España) ORCID: https://orcid.org/0000-0001-9019-2832 david@conecta13.com

\section{NOTA BIOGRÁFICA}

Licenciado en Matemáticas (Universidad de Granada) y Experto en e-Learning (Universidad de Sevilla). Docente en Enseñanza Secundaria (Matemáticas y Tecnología), coordinador pedagógico del Programa Andaluz de Alfabetización Digital y Acreditación en TICs de la Junta de Andalucía «aulae» y Jefe del Área de Gestión del Talento y Formación en el Consorcio Fernando de los Ríos. Socio fundador de Conecta13.

\section{RESUMEN}

La transformación digital de la administración pública y el perfil competencial del empleado público como consecuencia de esta transformación son dos de los temas de innovación en la administración pública más acuciantes y relevantes en este momento. El presente artículo acomete la realización de una revisión de la literatura con la intención de identificar los factores determinantes en relación con el proceso de transformación digital de la administración pública y del empleado público; junto a esta revisión, se realiza un estudio de casos cualitativo a través de la voz de personas expertas para profundizar en la definición de la transformación digital. Con esta información se propone la creación de un marco competencial para el empleado en una administración pública digital.

\section{PALABRAS CLAVE}

Transformación digital; administración pública; empleado público; innovación; tecnología. 


\begin{abstract}
The digital transformation of public administration and the competency profile of the public employee as a consequence of this transformation are two of the most pressing and relevant innovation issues in public administration at the moment. This article undertakes a literature review with the intention of identifying the determining factors in relation to the digital transformation process of the public administration and the public employee; together with this review, a qualitative case study is carried out through the voice of experts to deepen the definition of digital transformation. With this information, the creation of a competency framework for the employee in a digital public administration is proposed.
\end{abstract}

\title{
KEYWORDS
}

Digital transformation; public administration; civil employees; innovation; technology.

\section{SUMARIO}

1. DE LA TRANSFORMACIÓN DIGITAL DE LA ADMINISTRACIÓN PÚBLICA A LAS COMPETENCIAS DEL EMPLEADO PÚBLICO. 2. METODOLOGÍA DEL ESTUDIO. 3. REVISIÓN DE LA LITERATURA. 4. ANÁLISIS DE CASO: AGENTES CLAVE DE LA ADMINISTRACIÓN PÚBLICA. 5. CONCLUSIONES Y PROPUESTA DE MARCO COMPETENCIAL. REFERENCIAS BIBLIOGRÁFICAS.

\section{DE LA TRANSFORMACIÓN DIGITAL DE LA ADMINISTRACIÓN PÚBLICA A LAS COMPETENCIAS DEL EMPLEADO PÚBLICO ${ }^{1}$}

Vivimos una época de cambio acelerado, entre otras cuestiones por la tracción generada por las empresas tecnológicas y por los avances en investigación vinculada con la tecnología. Sin lugar a dudas, caminamos hacia una importante «transformación de la humanidad», como auguraba Schwab (2016), aunque no debemos dejar de ver que esta transformación avanza a distintas velocidades como consecuencia de desigualdades económicas y culturales que entrecruzan transversalmente nuestra sociedad.

En Administración Pública, la tecnología nos permite tener más información que nunca, compartirla con más facilidad, gestionarla y procesarla con más eficacia y prestar así un mejor servicio a la sociedad (Munuera Gómez, 2016); procedimientos que hace pocas décadas podían suponer horas de trabajo para distintas unidades posiblemente localizadas en diferentes ubicaciones hoy pueden ser realizados de manera instantánea, evitando desplazamientos y molestias al ciudadano, a través de la red.

Obviamente, la presencia de la tecnología en la administración pública no es un fenómeno novedoso sino en evolución. Se pueden distinguir tres fases en el proceso histórico de incorporación de la tecnología a la administración pública (Criado y Gil-García, 2019). En una primera fase centrada en la automatización de tareas y funciones, entre 1950 y 1970, la administración pública suplanta tecnológicamente ciertas actividades (por ejemplo, grandes cálculos) realizadas por humanos pero sin que esto represente un cambio en el funcionamiento interno de la administración.

En una segunda fase, entre 1980 y 2010, el despliegue de «microcomputadoras» en las organizaciones públicas permitió el rápido desarrollo y la difusión de las tecnologías de la información y la comunicación en el ámbito público. Con la llegada de la web e Internet se buscan la optimización de los procesos internos y la digitalización de los servicios, además de permitir la aparición de nuevas estructuras organizativas que permiten la interoperabilidad entre sistemas de diferentes organizaciones públicas.

Finalmente, la última fase hasta el momento, que transcurre desde 2010 hasta la actualidad, supone la transformación digital de la administración. El potencial de la tecnología transforma radicalmente el servicio público permitiendo una toma de decisiones más efectiva y basada en datos, dinámicas colaborativas entre grupos distribuidos por el territorio y en distintas unidades de la administración, mayor transparencia de los procesos, la transformación de las dinámicas de intermediación, la reducción de costes gracias a la accesibilidad de la información y una evaluación continua vinculada a la trazabilidad de las actuaciones de los nuevos espacios digitales de cooperación.

1 Los autores agradecen al equipo editorial de la revista la publicación de este artículo, así como los comentarios y sugerencias de los evaluadores anónimos que han mejorado su estructura y contenido. 
Además, el carácter transversal de las tecnologías digitales en las políticas de transformación de la administración pública es más que evidente en el contexto actual (Ballart y Ramió, 2000; Cortés Abad, 2020). Entre otras cuestiones, el paradigma del Gobierno Abierto (Salvador y Ramírez, 2016) o el reto de las smart cities (López-Fogués et al., 2017; Romero Tarín, 2018) ponen sobre la mesa la gestión a través de la tecnología de temas tan relevantes como la sostenibilidad y el medioambiente, la movilidad, la vivienda o la energía en el ámbito de la administración local.

Con todo este trasfondo, el Plan de Digitalización de las Administraciones Públicas 2021-2025 (Gobierno de España, 2021a) plantea tres ejes estratégicos: 1. Transformación digital de la Administración General del Estado; 2. Proyectos de alto impacto en la digitalización del Sector Público; y 3. Transformación digital y modernización del Ministerio de Política Territorial y Función Pública, Comunidades Autónomas y Entidades Locales. A su vez, dentro de este tercer eje se incluye la Medida 16, Transformación Digital y Modernización del Ministerio de Política Territorial y Función Pública, que propone lo siguiente:

La transformación digital de las Administraciones públicas prevista en la Agenda España 2025 pasa por que ésta llegue a todos los niveles de la Administración, incluyendo inversiones a nivel nacional, autonómico y local. En el ámbito del Ministerio de Política Territorial y Función Pública, y a través de esta medida, se abordan proyectos de modernización que mejorarán tanto los procesos y procedimientos administrativos como las competencias digitales y los recursos disponibles, para mejorar la atención a la ciudadanía y contribuir activamente al éxito de las políticas públicas destinadas a las grandes transformaciones de España (transformación digital, transformación medioambiental, cohesión social y cohesión territorial).

Así pues, la complejidad y la trascendencia de los cambios que genera la transformación digital requieren una revisión conceptual en profundidad para entender qué quiere decir realmente «transformación digital de la administración pública» y qué perfil de competencias se deriva para el empleado público a partir de este proceso de transformación.

En este sentido, el Plan Nacional de Competencias Digitales (Gobierno de España, 2021b) establece que:

la formación en competencias digitales para el empleo público posee una entidad separada y diferenciada del sector privado dado que es fundamental el desarrollo de las competencias digitales de los empleados y empleadas públicos, tanto las de carácter general como las del personal especializado en TIC, para que la Administración lleve a cabo una transformación interna (en procedimientos, relación con los administrados y cultura organizativa) que le permita desarrollar adecuadamente su papel como reguladora, impulsora y facilitadora de la transformación digital de España, y la formación en el ámbito de las Administraciones Públicas conforma un subsistema con características específicas, tal y como se recoge en la Ley 30/2015, de 9 de septiembre, por la que se regula el Sistema de Formación Profesional para el empleo en el ámbito laboral, y en el Acuerdo de Formación para el Empleo de las Administraciones Públicas (p. 34).

Para ello, el mencionado Plan propone un programa de capacitación digital de las Administraciones Públicas dentro del cual se debe realizar «un diagnóstico de situación en materia de capacitación digital de los empleados y empleadas públicos» y desarrollar y poner en marcha por parte del INAP un «plan de capacitación digital de las empleadas y empleados en la Administración General del Estado».

En resumen, con el horizonte creado por estos dos Planes del Gobierno de España, se plantea esta investigación con el objetivo de comprender, en toda su extensión, el proceso de transformación digital de la administración pública. Para ello el presente texto, un estudio de carácter mixto y exploratorio, se organiza en tres bloques: un primer apartado de revisión narrativa e integradora de la literatura; una segunda sección de entrevistas a personas expertas en administración pública digital para acotar, confirmar y contextualizar los hallazgos obtenidos en la primera fase. Con esta información se plantea, en las conclusiones del artículo, una propuesta de marco de competencias para el empleado público en el seno de la transformación digital de la administración.

\section{METODOLOGÍA DEL ESTUDIO}

El primer paso de este estudio es una revisión en profundidad de la literatura. En el ámbito de estudio de la Administración Pública y la tecnología existen referentes valiosos que pretenden abordar este complejo 
tema a través de revisiones de la literatura. Así, entre otros, Bouwman y Grimmelikhuijsen (2016) analizan la existencia de estudios experimentales en la Administración Pública entre 1992 y 2014; Marangunić y Granić (2015) revisan la aparición y el desarrollo del modelo de aceptación de la tecnología entre 1986 y 2013; Starke et al. (2021) analizan la «justicia algorítmica» en la toma de decisiones a través de sistemas de inteligencia artificial; finalmente, Zuiderwijk et al. (2021) estudiaron el uso de la inteligencia artificial en la gobernanza pública.

Snyder (2019) identifica tres tipos de revisión de la literatura: sistemática, narrativa e integradora. De estos tres planteamientos, la metodología integradora puede ser útil, según Snyder, «cuando el propósito de la revisión no es cubrir todos los artículos publicados sobre el tema sino combinar perspectivas para crear nuevos modelos teóricos» (p. 334). Al mismo tiempo, la revisión semi-sistemática o narrativa se utiliza cuando un tema ha sido estudiado desde diferentes perspectivas y por grupos muy variados de investigación que provienen de distintas disciplinas, haciendo imposible una revisión sistemática (Snyder, 2019, p. 335).

Así pues, desde la perspectiva de la revisión integradora y narrativa, la metodología a seguir en este estudio incluye los siguientes pasos:

TAbla 1. Metodología de la ReVisión de la literatura

\begin{tabular}{llll}
\hline \multicolumn{3}{c}{ Fase 1: Diseño de la revisión de la literatura } \\
\hline $\begin{array}{l}\text { Definición de las } \\
\text { estrategias de búsqueda } \\
\begin{array}{l}\text { más adecuadas para } \\
\text { el objeto de estudio. }\end{array}\end{array}$ & $\begin{array}{l}\text { Definición de los términos } \\
\text { de búsqueda. }\end{array}$ & $\begin{array}{l}\text { Selección de las bases } \\
\text { de datos de revistas } \\
\text { académicas. }\end{array}$ & $\begin{array}{l}\text { Determinación } \\
\text { de los criterios de inclusión } \\
\text { y exclusión. }\end{array}$ \\
\hline & Fase 2: Implementación de la revisión de la literatura \\
\hline $\begin{array}{l}\text { Documentación de los } \\
\text { procesos de búsqueda } \\
\text { y selección de artículos. }\end{array}$ & $\begin{array}{l}\text { Evaluación de la calidad } \\
\text { de los procesos de } \\
\text { búsqueda. }\end{array}$ & Análisis de la literatura. & $\begin{array}{l}\text { Síntesis de ideas y } \\
\text { triangulación de la síntesis } \\
\text { de los revisores }\end{array}$ \\
\hline
\end{tabular}

Fase 3: Redacción del informe

Fuente: Elaboración propia a partir de Snyder (2019). queda:

En este sentido, tras valorar distintas opciones se ha optado por utilizar la siguiente fórmula de bús-

ALL («digital transformation» OR «digital competence») AND «public administration» OR «civil servant» PUBYEAR > 2010 AND ( LIMIT-TO ( PUBSTAGE, «final») ) AND ( LIMIT-TO ( DOCTYPE, «ar») ) AND ( LIMIT-TO ( LANGUAGE, «English») OR LIMIT-TO ( LANGUAGE, «Spanish») ) AND ( LIMIT-TO ( SRCTYPE, «j») )

El horizonte de búsqueda incluye los años de 2010 a 2020. Entre los criterios de exclusión se consideran los siguientes:

- No se puede localizar el texto completo para su análisis.

- La muestra del artículo no se ajusta a los objetivos de esta investigación.

- El artículo no apunta a los objetivos de la investigación.

Los criterios de inclusión utilizados han sido los siguientes:

- Se puede localizar el texto completo para su análisis.

- El artículo trata sobre procesos de transformación digital en el ámbito de la administración pública.

- Se valorará si el artículo presenta una intervención y evidencias de resultados.

Para el análisis se utilizaron las bases de datos Web of Science y SCOPUS así como Google Scholar; en esta última web la búsqueda se limitó a las primeras veinte páginas de resultados siguiendo las indicaciones de Haddaway et al. (2015). Para el análisis de co-ocurrencias y la revisión de la literatura se ha usado el software Rayyan (Ouzzani et al., 2016). 
En una primera selección se detectaron 654 documentos que respondían a la fórmula de búsqueda. Tras analizar las co-ocurrencias, la muestra se limitó a 258 artículos recogidos en las bases de datos, que han sido los utilizados para la revisión. Para ello, dos revisores han analizado independientemente los artículos (título y abstract) y se ha realizado una selección final utilizando los criterios de inclusión y exclusión. Así pues, en total se han analizado 49 artículos de revistas científicas publicados entre 2010 y 2020, de los cuales se han seleccionado los artículos que conforman la presente revisión de la literatura por su particular incidencia en el objeto de estudio.

Figura 1. Diagrama de flujo del proceso de selección de la literatura

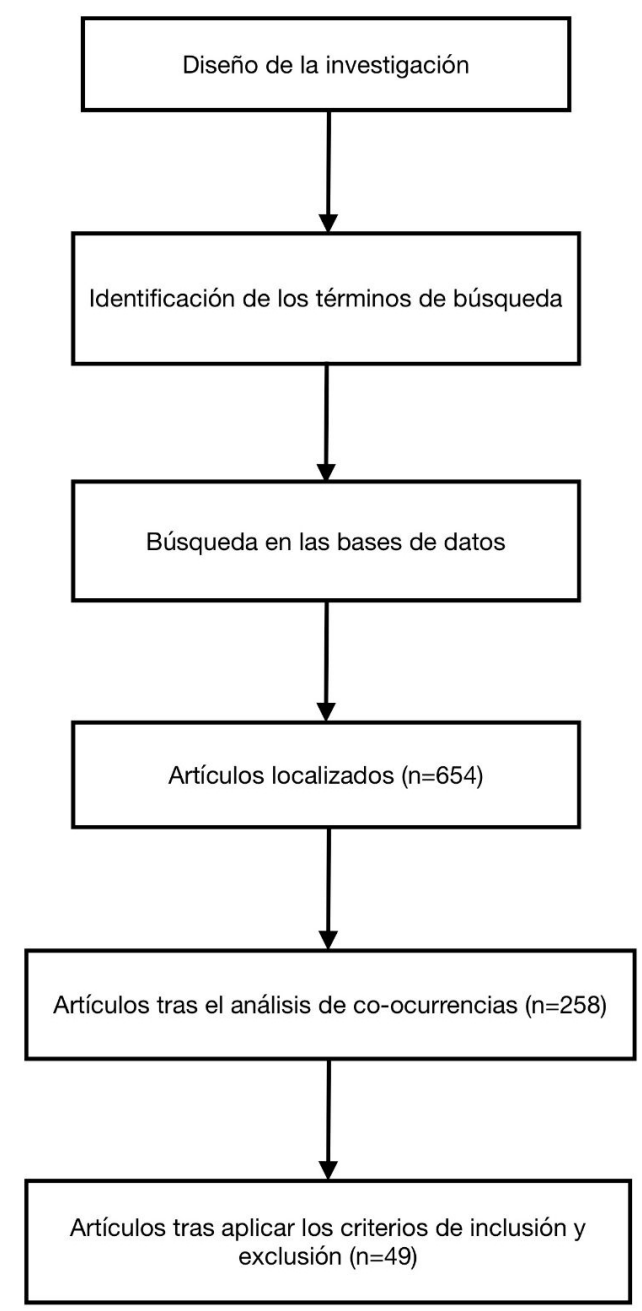

Fuente: Elaboración propia.

\section{REVISIÓN DE LA LITERATURA}

El primer objetivo en la revisión de la literatura ha sido poder definir el proceso de «transformación digital» como eje central de este estudio. Así, Vial (2019) define la transformación digital como «un proceso que aspira a mejorar una entidad promoviendo cambios significativos en sus propiedades a través de la combinación de tecnologías de la información, la computación, la comunicación y la conectividad» (p. 119). Es decir, la transformación digital es un proceso, marcado por fases y movimientos de avance y retroceso, cuyo objetivo es la mejora de una entidad (pública o privada) a través de cambios sustanciales de sus propiedades fundamentales mediante una combinación de diferentes tecnologías: tecnologías de la información, de la computación, de la comunicación y de la conectividad. 
En la misma línea, Mergel et al. (2019) definen la «transformación digital» mediante entrevistas con expertos. Para ello, especifican tres fases que marcan una secuencia en el proceso de transformación digital: en primer lugar, la «digitización», que representa la transición de servicios analógicos a digitales mediante un cambio de formato en los artefactos (en un lenguaje coloquial, «del papel al pdf») que se utilizan para prestar el servicio y el canal tecnológico para poder prestarlo; en segundo lugar, la «digitalización», que se centra en cambios en los procesos, implicando un movimiento de innovación ligada a la tecnología que redefine los procesos no sólo para que se desarrollen en el entorno digital sino para que sean más eficaces y adaptados al usuario; finalmente, la «transformación digital» representa un movimiento que incluye cambios culturales, organizativos y relacionales derivados del impacto de la tecnología en la administración.

Desde esa perspectiva, Mergel et al. (2019) definen la transformación digital como el esfuerzo global de revisar los procesos y los servicios prestados por la administración más allá del esfuerzo de «digitización», implicando un continuo de cambio desde lo analógico a lo digital gracias a un análisis de las necesidades de los usuarios, las políticas y los procesos, lo cual genera una revisión completa de los actuales servicios digitales y la creación de nuevos servicios. En resumen, esto supone una auténtica reinvención de la administración para amplificar algunos de sus valores definitorios: eficiencia, transparencia, rendición de cuentas, confianza y legitimidad.

TABla 2. CATEgorías del PROCeso de tRansformación digital de LA ADMinistración

\begin{tabular}{|c|c|c|c|}
\hline $\begin{array}{c}\text { Motivos para la } \\
\text { transformación digital }\end{array}$ & $\begin{array}{c}\text { Objetos de } \\
\text { transformación digital }\end{array}$ & $\begin{array}{c}\text { Procesos de } \\
\text { transformación digital }\end{array}$ & $\begin{array}{c}\text { Valor de la trasformación } \\
\text { digital }\end{array}$ \\
\hline $\begin{array}{l}\text { Razones endógenas } \\
\text { (necesidad de prestar un } \\
\text { mejor servicio) o exógenas } \\
\text { (extensión de los servicios } \\
\text { digitales en otros sectores } \\
\text { económicos o personales) }\end{array}$ & $\begin{array}{l}\text { Artefactos } \\
\text { Procesos } \\
\text { Organización } \\
\text { de la administración } \\
\text { Cultura burocrática }\end{array}$ & $\begin{array}{l}\text { Digitización } \\
\text { Cambio en las relaciones } \\
\text { Desarrollo } \\
\text { de competencias } \\
\text { Cambio en el marco } \\
\text { mental de empleados } \\
\text { públicos y usuarios }\end{array}$ & $\begin{array}{l}\text { Resultados a corto y largo } \\
\text { plazo } \\
\text { Impacto transformador. }\end{array}$ \\
\hline
\end{tabular}

Fuente: Elaboración propia a partir de Mergel et al. (2019).

En definitiva, como defienden Pittaway y Montazemi (2020), en el transcurso de la transformación digital se rediseñan los procesos organizativos fundamentales de la institución, se reemplazan las tecnologías obsoletas por otras nuevas, se desarrollan nuevas destrezas y se introducen nuevas maneras de trabajar que acaban transformando sustancialmente el funcionamiento de la propia institución.

Lee et al. (2018) explican que existen diversos motores para la transformación digital, entre los cuales destacan la acelerada transformación socio-técnica vinculada a las tecnologías inteligentes y el abordaje de problemas cada vez más complejos gracias a la configuración de los cinco elementos que aparecen en el núcleo de su propuesta: gobernanza, personas, servicios, datos y tecnología. Además, defienden que más allá del concepto de eficacia y mejora de la calidad del servicio público (véase Asgarkhani, 2005, para una revisión crítica del discurso de la «mejora»), el valor actual de la transformación digital de la administración es garantizar el desarrollo sostenible de la sociedad pues Lee et al. (2018) entienden la transformación digital como un marco de resolución de problemas y no simplemente como una organización peculiar de la administración.

En este sentido, Frennert (2019) estudia el concepto de transformación digital aplicado a la e-salud y la atención a las personas mayores o con discapacidad. En este contexto, presenta el concepto de «Tecnología del Bienestar» (welfare technology) para referirse al "conocimiento y uso de tecnología que permite mantener y/o aumentar el sentimiento de seguridad, actividad, participación e independencia de una persona (de cualquier edad) que tiene o ha visto aumentado el riesgo de tener/desarrollar una discapacidad» (p. 635). Es decir, la transformación digital de la administración puede generar «tecnologías del bienestar» que mejoren la vida de los ciudadanos y las ciudadanas.

En una línea similar, Barcevičius et al. (2019) revisan las principales aportaciones del gobierno y la administración electrónica en el contexto europeo. Señalan tres tipos de efectos de la aplicación de tecnologías digitales emergentes en la gestión pública: en primer lugar, ganancias en eficiencia y productividad que pueden contribuir a la reducción de costes operativos, la focalización de los empleados públicos en tareas 
esenciales e importantes y la prestación del servicio más rápida y barata; en segundo lugar, efectividad y mejoras en la calidad (predicciones más precisas, detección y trazabilidad en tiempo real de problemas, asignación eficaz de recursos, mejor toma de decisiones, servicios más personalizados y contextualizados y políticas y servicios más inclusivos y empoderadores); y finalmente, mayor transparencia, rendición de cuentas, confianza y legitimidad (aunque estos efectos son más difíciles de evaluar empíricamente).

Así pues, la tecnología sirve como detonante para el cambio y también es el medio por el cual se articula el cambio. Es decir, los procesos, las políticas, el liderazgo y los marcos mentales de las personas implicadas cambian para poder desarrollar plenamente la transformación digital al mismo tiempo que la introducción de la tecnología provoca cambios radicales en el funcionamiento de la administración y en las relaciones con la ciudadanía.

Vial (2019) describe este proceso mediante el ciclo de la transformación digital. La organización responde a disrupciones que están teniendo lugar en su entorno mediante una respuesta estratégica que incluye el uso de tecnología, lo cual alimenta la disrupción que originó el cambio y al mismo tiempo permite cambios en la creación de valor, en la cual inciden tanto barreras organizativas (inercias y resistencias) como cambios estructurales (cultura y estructura organizativa, liderazgo, roles y destrezas de los trabajadores). Finalmente, este conglomerado de cambios genera impactos positivos (relacionados con la eficiencia de las operaciones, el desempeño de la organización, mejoras sociales y en el tejido industrial) y también negativos (seguridad y privacidad).

Para entender de qué tipo de tecnología estamos hablando como palancas de la transformación digital, Pereira et al. (2020) destacan que en este momento los cuatro pilares tecnológicos de la transformación digital son la computación en la nube, la tecnología móvil, la tecnología social y la analítica de datos. Estos cuatro pilares se alimentan, a su vez, de una serie de soluciones que han servido como «aceleradores de innovación», entre las cuales se incluyen el Internet de las cosas (IOT), la robótica, la impresión 3D, el blockchain, la inteligencia artificial, la realidad aumentada y virtual, los sistemas cognitivos y la seguridad de última generación. En la misma línea, Barcevičius et al. (2019) analizan, entre las tecnologías digitales emergentes que tienen un impacto importante en la administración pública, la inteligencia artificial, la analítica del comportamiento y la analítica predictiva, la robótica y la automatización, el Internet de las cosas, la explotación de datos geoespaciales y de geolocalización, el blockchain y los interfaces de datos de gobierno abierto y de programación de aplicaciones. Por último, Casalino et al. (2020, p. 323) identifican dieciocho temas a considerar en la transformación digital de la administración pública:

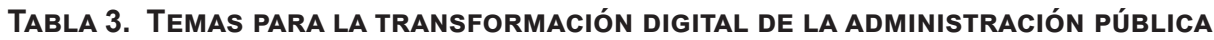

\begin{tabular}{lll}
\hline \multicolumn{1}{c}{$\begin{array}{c}\text { Digitalización de procesos } \\
\text { operativos }\end{array}$} & $\begin{array}{c}\text { Gobernanza de la digitalización } \\
\text { en el sector público }\end{array}$ & \multicolumn{1}{c}{$\begin{array}{c}\text { Integración digital de servicios } \\
\text { en línea y activos de producción }\end{array}$} \\
\hline $\begin{array}{l}\text { Sistemas de gestión documental } \\
\text { y digitalización del flujo de trabajo }\end{array}$ & $\begin{array}{l}\text { Rediseño de los sistemas clásicos } \\
\text { de información (cloud computing, } \\
\text { edge computing, distributed ledger } \\
\text { technologies) }\end{array}$ & $\begin{array}{l}\text { Gestión, integración y análisis } \\
\text { de datos }\end{array}$ \\
\hline Gestión de big data & Internet de las Cosas (loT) & Interacción humano-máquina \\
\hline Realidad virtual y aumentada & $\begin{array}{l}\text { Manufactura aditiva (impresión 3D, } \\
\text { Rapid Prototyping, Direct Digital } \\
\text { Manufacturing, etc.) }\end{array}$ & $\begin{array}{l}\text { Robótica avanzada y colaboración } \\
\text { humano-robot }\end{array}$ \\
\hline $\begin{array}{l}\text { Ahorro de energía e impacto } \\
\text { medioambiental }\end{array}$ & $\begin{array}{l}\text { Ineficiencia digital y riesgos en el } \\
\text { entorno laboral }\end{array}$ & Sistemas de automatización \\
\hline $\begin{array}{l}\text { Protección de datos y privacidad } \\
\text { en organizaciones públicas }\end{array}$ & $\begin{array}{l}\text { Inteligencia Artificial y sistemas } \\
\text { expertos }\end{array}$ & $\begin{array}{l}\text { Certificación de la cadena } \\
\text { de provisión y verificación } \\
\text { automática de procesos }\end{array}$ \\
\hline
\end{tabular}

Fuente: Elaboración propia a partir de Casalino et al. (2020).

En el plano organizativo, Zhang et al. (2014) apuntan cuatro grandes categorías: en primer lugar, la estructura de la propia administración (tipo de gobernanza, tamaño y estructura de las unidades, recursos, 
colaboración inter-institucional y relaciones público-privadas); en segundo lugar, el papel de los empleados públicos (constructos y marcos mentales que manejan los empleados públicos, destrezas y redes de atención interorganizativa); en tercer lugar, el liderazgo, que favorece la emulación de las unidades que hayan experimentado una transformación exitosa, la resolución de problemas y la búsqueda de recursos; y, finalmente, la distribución de poder, especialmente si este está descentralizado.

Por último, Zhang et al. (2014) señalan tres tipos de factores para la difusión de la administración electrónica: factores tecnológicos, organizativos y del entorno. En cuanto a los factores «ambientales», podemos analizarlos en tres planos: factores macro-ambientales como la cultura o la política; factores meso-ambientales, como el tamaño de la unidad administrativa (país, región o ciudad); y factores micro-ambientales vinculados con el entorno cultural y organizativo de la administración.

Así pues, como afirman Gil-García et al. (2018) el «gobierno digital» es un fenómeno que implica nuevos estilos de liderazgo, nuevos procesos de toma de decisiones, diferentes maneras de organizar y prestar el servicio público e incluso un nuevo concepto de ciudadanía. Al mismo tiempo, detectan en este ámbito de estudio una serie de rasgos propios de la administración y el servidor público inmerso en la transformación digital y que van más allá de la relación entre tecnología y eficacia/efectividad, como son liderazgo, confianza, transparencia, participación o gestión colaborativa de redes de datos.

Castelnovo y Sorrentino (2018) advierten de la dificultad de gestionar los «múltiples factores difíciles de gobernar» de los cuales depende la transformación digital y destacan tres aspectos cruciales en la implementación de la administración digital: el diseño de los planes de coordinación, la distribución de competencias y responsabilidades entre múltiples actores y la capacidad de cada unidad administrativa de adoptar las estrategias de cambio organizativo para traducir el potencial de los recursos tecnológicos en servicios potenciales. Al mismo tiempo, recuerdan que la tecnología no transforma por sí misma a la administración o el gobierno, como también defiende Asgarkhani (2005), sino que amplifica tendencias políticas existentes o emergentes, las cuales, a su vez, dependen de variables contextuales (estructurales-instrumentales, culturales y ambientales) específicas del país o la región que es objeto de análisis.

Por otro lado, Clarke (2020) analiza el funcionamiento de «unidades de gobierno digital». El sentido de estas unidades es representar «unidades internas de competencia y experiencia digital en el centro del gobierno, frente a modelos vinculados a departamentos específicos» con el objetivo de revertir «deficiencias bien documentadas en la gobernanza TIC del sector público y, de manera más ambiciosa, para transformar las prácticas de gestión pública en general» (p. 361).

Clarke (2020) define los rasgos de estas unidades, que representan también rasgos de la nueva ortodoxia digital: el desarrollo ágil y centrado en el usuario, los procesos iterativos y basados en prototipos, con un peso importante de los datos en el proceso de toma de decisiones y diseño, con estándares abiertos y un enfoque basado en plataformas que rompe con los silos departamentales, tanto desde la perspectiva de la arquitectura de red como en relación con las culturas burocráticas que estos mantienen, funcionando de manera más parecida a una start-up como unidad de innovación que a una unidad tradicional de la administración (incluyendo entre las diferencias también los modos de selección del personal y los perfiles requeridos).

Obviamente, todos estos cambios tienen un impacto en el trabajo del empleado público. Colbert et al. (2016) sentencian que «la fuerza laboral digital ha desarrollado muchas competencias en el transcurso de sus interacciones con la tecnología que podrían servir de palanca en el puesto de trabajo» (p. 732). Entre estas competencias destaca la «fluidez digital», que implica dominio y comodidad en el uso de programas o aplicaciones básicas y en la gestión de la información, la creación de ideas y el uso de la tecnología para conseguir metas estratégicas. Finalmente, Colbert et al. también señalan competencias como las destrezas de liderazgo, la asunción de riesgos y el aprendizaje a partir de la experiencia y los errores.

Por otro lado, la prevalencia de la tecnología en la vida de los trabajadores (públicos o privados) conlleva cambios en el desarrollo y la expresión de la identidad, las relaciones interpersonales y la capacidad de colaboración. Así, en el plano identitario se observa una cierta tensión entre la autoconciencia y la autorepresentación en los social media. Al mismo tiempo, prácticas como el texting han cambiado nuestras expectativas respecto a las relaciones interpersonales y qué podemos esperar de ellas, con cambios reconocibles en valores tan importantes como la empatía.

Una postura similar adopta el Foro Económico Mundial (World Economic Forum [WEF], 2015) cuando analiza el potencial de la tecnología desde la perspectiva del desarrollo del individuo. Así, se definen dieciséis habilidades del siglo XXI, que se organizan en tres campos: alfabetizaciones fundamentales, competencias y cualidades del individuo: 
GAPP. Nueva Época - N. 27, noviembre 2021 - ISSN: 1989-8991 - DOI: https://doi.org/10.24965/gapp.i27.10923 - [Págs. 49-67]

Transformación digital de la administración pública: ¿Qué competencias necesitan los empleados públicos? Fernando Trujillo Sáez / David Álvarez Jiménez

TABla 4. Habilidades del SIGlo XXI

\begin{tabular}{|c|c|c|c|c|c|}
\hline \multicolumn{6}{|c|}{ Alfabetizaciones fundamentales } \\
\hline $\begin{array}{l}\text { Alfabetización y } \\
\text { Aritmética }\end{array}$ & $\begin{array}{l}\text { Alfabetización } \\
\text { Científica }\end{array}$ & Alfabetización TIC & $\begin{array}{l}\text { Alfabetización } \\
\text { Financiera }\end{array}$ & $\begin{array}{l}\text { Alfabetización } \\
\text { Cultural }\end{array}$ & $\begin{array}{l}\text { Alfabetización } \\
\text { Cívica }\end{array}$ \\
\hline \multicolumn{6}{|c|}{ Competencias } \\
\hline $\begin{array}{l}\text { Pensamiento } \\
\text { Crítico }\end{array}$ & Resolución de & roblemas & Creatividad & Comunicación & Colaboración \\
\hline \multicolumn{6}{|c|}{ Cualidades del individuo } \\
\hline Curiosidad & Iniciativa & Persistencia y Valor & Adaptabilidad & Liderazgo & $\begin{array}{l}\text { Conciencia } \\
\text { Social y Cultural }\end{array}$ \\
\hline
\end{tabular}

Fuente: Elaboración propia a partir de World Economic Forum, 2015.

Por su parte, el Consejo de Europa (Comisión Europea [CE], 2019) establece un conjunto de competencias clave con el objetivo de adaptarse a la realidad cambiante de un mundo interconectado donde cada persona necesita (o necesitará) un amplio rango de habilidades y de competencias que deberá desarrollar continuamente a lo largo de su vida. En este sentido, las ocho competencias que establece este marco son:

1. Competencia de/en alfabetización.

2. Competencia multilingüe.

3. Competencia matemática y competencia en ciencia, tecnología e ingeniería.

4. Competencia digital.

5. Competencia personal, social y de aprender a aprender.

6. Competencia ciudadana.

7. Competencia emprendedora.

8. Competencia de conciencia y expresión cultural.

Además, habilidades tales como pensamiento crítico, resolución de problemas, trabajo en equipo, habilidades de comunicación y negociación, habilidades analíticas, creatividad y habilidades interculturales están integradas en todas las competencias clave.

En línea con estos documentos, el Joint Research Centre (Sala et al., 2020) ha desarrollado un marco europeo para la competencia personal, social y de aprender a aprender, una competencia clave que se entiende que es el umbral de muchos otros aprendizajes a lo largo de la vida. En este sentido, para el análisis de esta competencia se hace uso de conceptos que cubren ámbitos como las life skills, las soft skills, las competencias socioemocionales, las habilidades no cognitivas, las competencias transversales, las competencias del siglo XXI y las competencias 2030.

Desde la perspectiva de la competencia digital también contamos con marcos de referencia que pueden servir a nuestra tarea. Un documento destacado es el Marco de Competencia Digital para la Ciudadanía (Carretero et al., 2017), que define cinco competencias organizadas en cuatro niveles de dominio (foundation, intermediate, advanced y highly specialised):

Tabla 5. Marco de Competencia Digital para la Ciudadanía

Información y alfabetización informacional

Identificar, localizar, recuperar, almacenar, organizar y analizar la información digital, evaluando su finalidad y relevancia.

\section{Comunicación y colaboración}

Comunicar en entornos digitales, compartir recursos a través de herramientas en línea, conectar y colaborar con otros a través de herramientas digitales, interactuar y participar en comunidades y redes; conciencia intercultural. 
GAPP. Nueva Época - N. 27, noviembre 2021 - ISSN: 1989-8991 - DOI: https://doi.org/10.24965/gapp.i27.10923 - [Págs. 49-67]

Transformación digital de la administración pública: ¿Qué competencias necesitan los empleados públicos?

Fernando Trujillo Sáez / David Álvarez Jiménez

\section{Creación de contenido digital}

Crear y editar contenidos nuevos (textos, imágenes, videos...), integrar y reelaborar conocimientos y contenidos previos, realizar producciones artísticas, contenidos multimedia y programación informática, saber aplicar los derechos de propiedad intelectual y las licencias de uso.

\section{Seguridad}

Protección personal, protección de datos, protección de la identidad digital, uso de seguridad, uso seguro y sostenible.

\section{Resolución de problemas}

Identificar necesidades y recursos digitales, tomar decisiones a la hora de elegir la herramienta digital apropiada, acorde a la finalidad o necesidad, resolver problemas conceptuales a través de medios digitales, resolver problemas técnicos, uso creativo de la tecnología, actualizar la competencia propia y la de otros.

Fuente: Elaboración propia a partir de Carretero et al. (2017).

Además, a partir de este documento se han desarrollado otros marcos, como el Marco UNESCO (Law et al., 2018) de referencia en habilidades de alfabetización digital para el indicador 4.4.2. de los Objetivos de Desarrollo Sostenibles:

TABLA 6. MARCo UNESCO de REFERENCIA EN HABILIDADES de ALFABETIZACIÓN DigitAL PARA EL INDICADOR 4.4.2. de los ObJetivos de Desarrollo Sostenibles

Dispositivos y operaciones de software

\begin{tabular}{|c|c|c|c|}
\hline \multicolumn{2}{|c|}{ Operaciones físicas de dispositivos digitales } & \multicolumn{2}{|c|}{ Operaciones de software en dispositivos digitales } \\
\hline \multicolumn{4}{|c|}{ Alfabetización informacional y de datos } \\
\hline $\begin{array}{l}\text { Navegar, buscar y filtrar da } \\
\text { digital. }\end{array}$ & s, información y contenido & $\begin{array}{l}\text { Evaluar datos, información } \\
\text { y contenido digital. }\end{array}$ & $\begin{array}{l}\text { Manejar datos, información } \\
\text { y contenido digital. }\end{array}$ \\
\hline \multicolumn{4}{|c|}{ Comunicación y colaboración } \\
\hline $\begin{array}{l}\text { Interactuar a través de las } \\
\text { tecnologías digitales }\end{array}$ & $\begin{array}{l}\text { Compartir a través de las } \\
\text { tecnologías digitales }\end{array}$ & $\begin{array}{l}\text { Comprometerse/participar } \\
\text { en ciudadanía a través de } \\
\text { las tecnologías digitales }\end{array}$ & $\begin{array}{l}\text { Colaborar a través de las } \\
\text { tecnologías digitales }\end{array}$ \\
\hline Net-Etiqueta & & Manejar la identidad digital & \\
\hline \multicolumn{4}{|c|}{ Creación de contenidos digitales } \\
\hline $\begin{array}{l}\text { Desarrollar contenido } \\
\text { digital }\end{array}$ & $\begin{array}{l}\text { Integrar y reelaborar } \\
\text { contenido digital }\end{array}$ & $\begin{array}{l}\text { Entender y usar copyrights } \\
\text { y licencias }\end{array}$ & Programar \\
\hline \multicolumn{4}{|c|}{ Seguridad } \\
\hline Proteger los dispositivos & $\begin{array}{l}\text { Proteger datos personales } \\
\text { y privacidad }\end{array}$ & $\begin{array}{l}\text { Proteger la salud y el } \\
\text { bienestar }\end{array}$ & Proteger el entorno \\
\hline \multicolumn{4}{|c|}{ Resolución de problemas } \\
\hline $\begin{array}{l}\text { Resolver problemas } \\
\text { técnicos }\end{array}$ & $\begin{array}{l}\text { Usar pensamiento } \\
\text { computacional }\end{array}$ & $\begin{array}{l}\text { Usar creativamente las } \\
\text { tecnologías digitales }\end{array}$ & $\begin{array}{l}\text { Identificar brechas de } \\
\text { competencia digital }\end{array}$ \\
\hline \multicolumn{4}{|c|}{ Identificar necesidades y respuestas tecnológicas } \\
\hline \multicolumn{4}{|c|}{ Competencias relacionadas con la carrera profesional } \\
\hline $\begin{array}{l}\text { Operar tecnologías especia } \\
\text { particular }\end{array}$ & zadas para un campo & $\begin{array}{l}\text { Interpretar y manipular dato } \\
\text { digital para un campo concr }\end{array}$ & $\begin{array}{l}\text {, información y contenido } \\
\text { to }\end{array}$ \\
\hline
\end{tabular}

Fuente: Elaboración propia a partir de Law et al. (2018). 
De manera específica en relación con los empleados públicos, Kruyen y Van Genugten (2020) señalan que la mayor parte de los textos que tratan las competencias del servidor público son claramente normativos y predominantemente orientados hacia las competencias de gestión del alto funcionariado; además observan en los marcos de referencia la dificultad de delimitar si las competencias que se presentan están vinculadas con diferentes maneras de entender la función pública: en concreto, desde los principios de la administración pública tradicional, desde los principios del New Public Management (vigente desde los años ochenta hasta la actualidad) o a la filosofía más reciente de la Nueva Gobernanza Pública (New Public Governance).

Para evitar estos problemas, Kruyen y Van Genugten investigan la opinión de empleados públicos acerca de las competencias necesarias hoy para realizar su trabajo. Estas competencias son agrupadas en una serie de clusters no excluyentes:

- Competencias de la administración pública tradicional: expertise, conocimiento y destrezas jurídicas, destrezas administrativas, independencia, atención a los detalles y sensibilidad a las diferencias políticas.

- Competencias de la Nueva Gestión Pública: eficacia, gestión del tiempo, orientado a los resultados, gestión de proyectos, trabajo basado en proyectos, visión táctica y capacidad de control y de rendición de cuentas.

- Competencias de la Nueva Gobernanza Pública: colaboración, trabajo en red, co-creación, destrezas sociales y comunicativas, orientado a las personas, destrezas de negociación y arbitraje, improvisación, gestión de las expectativas, visión estratégica, competencia intercultural y de atención a la diversidad, capacidad de escucha, empatía y competencia medioambiental.

- Competencias cognitivas: destrezas analíticas, juicio y visión global.

- Integridad: fiabilidad, incorruptibilidad, confianza y honestidad.

- Creatividad: capacidad de innovación, capacidad de creación de conexiones cognitivas, flexibilidad, atrevimiento, capacidad emprendedora, actitud proactiva, comprensión del entorno, apertura mental e inconformismo.

- Resolución (Getting things done): planificación y organización, gestión de las resistencias, compromiso, perseverancia, capacidad de priorizar, resistencia al estrés, asertividad, pragmatismo y realismo.

- Comunicación y persuasión: destrezas comunicativas, habilidades conversacionales, habilidades lingüísticas, habilidades para la argumentación y el debate, capacidad para convencer y emocionar.

- Liderazgo: liderazgo en grupos, coaching, liderazgo transformador, liderazgo colaborativo, capacidad para motivar, visión de futuro y capacidad para delegar.

- Desarrollo personal: capacidad para aprender, auto-gestión y auto-conocimiento.

En la misma línea de generación de agrupaciones de destrezas, OCDE $(2017$, p. 32) determina cuatro conjuntos de destrezas: policy advisory skills, engagement skills, commissioning skills y networking management skills. En estas destrezas la tecnología juega un papel transversal que conlleva la necesaria capacitación del empleado público (OCDE, 2017):

A pesar de que todo el funcionariado no necesitará ser especialista digital, sí necesitarán entender el potencial ( $\mathrm{y}$ los riesgos asociados) de las nuevas herramientas tecnológicas y desarrollar destrezas complementarias a estas nuevas herramientas para implicarse con los ciudadanos, analizando los problemas de las políticas y sirviendo de palanca para las oportunidades digitales (p. 17).

Así pues, una administración digital puede convertirse en una palanca de transformación para la sociedad y para el propio empleado público, que a su vez es el principal agente para la puesta en práctica de la administración digital y para la creación de valor a partir de la transformación digital de la misma.

En este sentido, las competencias del empleado público en una administración digital (o en proceso de transformación digital) trascienden la mera competencia digital. En primer lugar, el empleado público requiere una competencia para el aprendizaje permanente que le permita tener control sobre su propio aprendizaje y hacer de este una herramienta para la actualización de conocimientos y para su desarrollo.

Así mismo, el empleado público es una persona que, gracias a su competencia para el aprendizaje permanente, se encuentra en desarrollo en tres planos: desarrollo personal, desarrollo ciudadano y desarrollo profesional. Así pues, el servidor público, que no sólo trabaja en la administración pública sino que vive en 
un entorno y en una sociedad determinados, pone al servicio de su papel como empleado público no sólo las destrezas estrictamente vinculadas con el desempeño de su puesto de trabajo sino todos sus conocimientos y competencias como persona, ciudadano y profesional.

Finalmente, la transformación digital requiere del empleado público también una serie de competencias vinculadas directamente con la tecnología y los recursos digitales. En concreto, las competencias para la transformación digital demandan el uso de dispositivos, software, plataformas y redes; la alfabetización informacional y de datos; la comunicación y colaboración en entornos digitales; la creación de contenido digital; la búsqueda y garantía de seguridad en entornos digitales; y la resolución de problemas con y acerca de la tecnología.

\section{ANÁLISIS DE CASO: AGENTES CLAVE DE LA ADMINISTRACIÓN PÚBLICA}

La contextualización y triangulación del conocimiento proporcionado por la revisión de la literatura es una condición primordial para ajustar el producto final a las condiciones reales de uso. Para ello se plantea la realización de entrevistas a stakeholders de la administración pública vinculados con la transformación digital así como con expertos en transformación digital de la administración pública.

Döringer (2020) introduce el concepto de agentes clave como participantes privilegiados en una investigación como la que nos ocupa. Estos agentes tienen un papel fundamental en la toma de decisiones y el seguimiento del proceso de cambio puesto que de sus marcos mentales depende el marco cultural en el cual tiene lugar el proceso de transformación. En este sentido, se consideran informantes fundamentales para la definición de un proceso siempre cambiante y con momentos de evolución e involución.

Para realizar las entrevistas a estos agentes clave, Döringer (2020) propone la siguiente secuencia:

El entrevistador comienza con una pregunta abierta para estimular una narración estructurada a partir de las preocupaciones del individuo. Una vez que el entrevistado ha finalizado el episodio narrativo, el entrevistador se mueve a exploraciones generales y específicas así como preguntas ad hoc. A través de exploraciones generales, los investigadores intentan inquirir aspectos y detalles que son de interés potencial para la investigación pero que no hayan sido mencionados por el entrevistado aún. Por otro lado, las exploraciones específicas se refieren directamente a la explicación del entrevistado para ganar una mayor comprensión de sus opiniones (p. 268).

En este estudio exploratorio y de confirmación de hipótesis emanadas de la revisión de la literatura se han realizado en total cinco entrevistas en profundidad: tres entrevistas con responsables autonómicos de la administración pública (directores generales vinculados con procesos de transformación digital) y dos entrevistas con sendas expertas en transformación digital de la administración pública, ambas funcionarias. Las comunidades autónomas participantes en la investigación son Castilla y León, Cataluña y Andalucía.

Estos «agentes clave» han sido elegidos por nominación entre iguales a partir de informantes de la Administración Pública familiarizados con la temática de esta investigación (Bankins et al., 2017). Las entrevistas se han realizado utilizando la herramienta Google Meet. Todas las entrevistas han sido grabadas tras la lectura y firma del consentimiento informado donde se explicaban los objetivos y la ética de la investigación. Tras la grabación de las entrevistas, estas han sido transcritas y analizadas con el software MAXQDA 2020. Las entrevistas han sido anonimizadas para garantizar la confidencialidad de los informantes.

Tras el análisis de las entrevistas, el primer mensaje de los participantes es que la transformación digital es un proceso complejo y que, a pesar de parecer reciente, lleva sin embargo años teniendo lugar de manera paralela al desarrollo tecnológico de la industria y la propia sociedad. Así pues, se acumulan en torno a este concepto muchos significados que es necesario ordenar para tener una visión clara de qué significa y de cómo se puede promover de manera eficaz.

A partir del análisis de las entrevistas surgen diversas categorías que nos permiten entender mejor el proceso de transformación digital y cómo puede promoverse. Las categorías inferidas del discurso de los agentes clave son las siguientes:

- Palancas de cambio.

- Vectores de cambio del empleado público.

- Transformaciones estructurales.

- Vías de transformación.

- Desarrollos pendientes. 
Para cada una de estas categorías ofrecemos a continuación un análisis de los nodos y significados que emanan de las entrevistas.

El proceso de transformación digital se basa en la puesta en funcionamiento de una serie de «palancas de cambio». Entre estas palancas surgen cinco conceptos clave: sociedad, personas, procesos, tecnologías y datos. Los cambios producidos en cada uno de estos planos así como la interacción entre los mismos suponen el auténtico motor para la transformación digital. Esta idea queda bien reflejada en una expresión repetida por todos los agentes entrevistados: en la sociedad actual se está viendo un proceso de transformación digital y las administraciones públicas deben ir a la misma velocidad que evoluciona el resto de la sociedad.

Además, estas «palancas de cambio» generan un movimiento con un sentido específico en el caso de la administración pública. Como afirmaba uno de los informantes, la transformación digital va dirigida a mejorar la misión de la administración pública, que consiste en «prestar servicios públicos gratuitos, defender el interés y los bienes comunes y mejorar la calidad de vida de las personas, especialmente aquellos que tienen menos recursos y cuyo punto de partida es peor».

Una segunda categoría importante son los «vectores de cambio del empleado público», que da cabida a cuestiones como las siguientes funciones o rasgos:

- Mentalidad y cultura organizativa ajustadas a la nueva realidad de una administración digital.

- Modernización del servicio público.

- Transparencia reforzada de la administración pública a través de los servicios digitales.

- Flexibilidad como competencia profesional y también como actitud vital.

- Innovación para diseñar un servicio proactivo en lugar de un servicio reactivo.

- Apropiación (o cultura) digital, que garantiza no sólo el dominio de las herramientas tecnológicas sino una predisposición al aprendizaje tecnológico permanente para estar al día de los cambios tecno-sociales que ocurren a nuestro alrededor.

- Pensamiento crítico ante la información y el desarrollo tecnológico.

- Compromiso y competencia social para no perder de vista que trabajamos dentro de un sistema social y cada uno con una identidad social y digital determinada.

- Resiliencia para estar preparados para los problemas y los riesgos que pueden surgir, especialmente en un contexto de teletrabajo.

- Colaboración para trabajar en red y en un modo social y colaborativo.

- Capacitación y formación incluyendo aprendizajes formales e informales, así como procesos de autoformación.

- Orientación al dato para la toma de decisiones y el diseño informado.

- Sostenibilidad (pensamiento ecológico, uso de software libre, etc.).

La categoría «transformaciones estructurales» recoge aquellos cambios que, aunque afectan al empleado público, en realidad dependen de la propia arquitectura de la administración digital. En primer lugar, es claramente visible el deseo de poner al ciudadano en el centro de la administración digital, pensando los procedimientos desde la perspectiva del ciudadano y no de la norma o de la propia administración. Así, por ejemplo, es necesario pensar en colectivos concretos que pueden tener problemas para el manejo en la administración digital, como las personas de mayor edad o las personas que residen en zonas rurales con poca cobertura digital. Derivada de esta preocupación surge un «desarrollo pendiente» importante: la formación de la ciudadanía para la administración digital, fundamental para superar la brecha digital y para garantizar la igualdad de los ciudadanos ante la administración pública.

Para poder atender eficazmente a esta diversidad de ciudadanos se recoge, también con mucha claridad, una llamada a la simplificación de los procedimientos de la administración digital, en buena medida por comparación con otros procedimientos utilizados para relacionarnos con empresas de la industria digital: como afirma uno de los participantes en nuestra investigación, «si comprar en Amazon o relacionarte con el banco es sencillo, la Administración digital tiene que ser tan sencilla como estos procesos». Esta simplificación es especialmente necesaria, según los informantes, en relación con la gestión de los expedientes y las herramientas necesarias para esta gestión.

Al mismo tiempo, de cara al empleado público, es necesario invertir en herramientas digitales, plataformas y redes que faciliten el desempeño de su trabajo -o incluso teletrabajo, como se ha experimentado durante el confinamiento provocado por el COVID-19-. La existencia de estas herramientas, plataformas y redes de trabajo requieren formación y una nueva manera de entender el trabajo dentro de la administración, 
superando la visión por «silos» o departamentos. En este sentido, será necesario superar una doble resistencia al cambio: el paso de procedimientos analógicos a digitales y el cambio de mentalidad para el trabajo colaborativo en red.

Por otro lado, la categoría «vías de transformación» contempla cuestiones como la capacitación, el empoderamiento del empleado público y la revisión de los procesos de selección. En este sentido, se entiende que la certificación de la competencia digital puede ser un buen camino para apoyar la transformación digital de la administración y el empleado público desde la formación y el autoconocimiento del individuo. Así mismo, destaca la importancia del liderazgo para promover y hacer sostenible el proceso de transformación digital, con sus avances y sus retrocesos. Como afirmaba una de las informantes, «los responsables de las organizaciones son fundamentales en el impulso de la transformación, porque su actitud determina los cambios que sus organizaciones pongan en marcha». Se necesita, por tanto, un liderazgo claro y sin ambivalencias para apoyar la transformación digital de la administración.

Finalmente, la categoría «desarrollos pendientes» alberga aquellas cuestiones que aún no han sido implementadas totalmente en la administración pública y que, por tanto, requieren mayor apoyo y atención. Dentro de esta categoría se encuentran cuestiones como la orientación al dato, la ciberseguridad, la ciberresiliencia, la apropiación (o cultura) digital o la innovación. Muchos de estas cuestiones están vinculadas con una forma diferente de entender la administración pública ya que, como afirma una de las informantes, «no es simplemente una cuestión de uso de herramientas digitales sino de cambio de actitud respecto a cómo se gestionan los servicios públicos».

Por último, entre estos «desarrollos pendientes» está la vinculación del desarrollo personal del empleado público y su desarrollo profesional. Ambas facetas, como decía una de las expertas entrevistadas, avanzan juntas («es muy difícil separar el desarrollo personal del profesional») y suponen una visión holística del empleado público como individuo, como profesional y como ciudadano que aborda la transformación digital de su entorno desde la voluntad y la necesidad del aprendizaje permanente a lo largo de la vida.

Tabla 7. Claves de la transformación digital de la Administración Pública

\begin{tabular}{|c|c|}
\hline \multirow[t]{5}{*}{ Palancas de cambio } & Sociedad \\
\hline & Personas \\
\hline & Procesos \\
\hline & Tecnologías \\
\hline & Datos \\
\hline \multirow[t]{13}{*}{ Vectores de cambio del empleado público } & Mentalidad y cultura organizativa digital \\
\hline & Modernización del servicio público \\
\hline & Transparencia reforzada \\
\hline & Flexibilidad \\
\hline & Innovación \\
\hline & Apropiación (o cultura) digital \\
\hline & Pensamiento crítico \\
\hline & Compromiso y competencia social \\
\hline & Resiliencia \\
\hline & Colaboración \\
\hline & Capacitación y formación \\
\hline & Orientación al dato \\
\hline & Utilización de software libre \\
\hline
\end{tabular}


GAPP. Nueva Época - N. 27, noviembre 2021 - ISSN: 1989-8991 - DOI: https://doi.org/10.24965/gapp.i27.10923 - [Págs. 49-67]

Transformación digital de la administración pública: ¿Qué competencias necesitan los empleados públicos?

Fernando Trujillo Sáez / David Álvarez Jiménez

\begin{tabular}{l|l}
\hline \multirow{2}{*}{ Transformaciones estructurales } & El ciudadano en el centro de la administración digital \\
\cline { 2 - 2 } & Simplificación de los procedimientos \\
\cline { 2 - 2 } Vías de transformación & Inversión en herramientas digitales \\
\hline \multirow{2}{*}{ Desarrollos pendientes } & $\begin{array}{l}\text { Capacitación, empoderamiento y certificación de la competencia } \\
\text { digital del empleado público }\end{array}$ \\
\cline { 2 - 2 } & Revisión de los procesos de selección \\
\cline { 2 - 2 } & Liderazgo del empleado público \\
\hline & Orientación al dato \\
\cline { 2 - 2 } & Ciberseguridad \\
\cline { 2 - 2 } & Ciber-resiliencia \\
\cline { 2 - 2 } & Apropiación (o cultura) digital \\
\cline { 2 - 2 } & Innovación digital \\
\hline
\end{tabular}

Fuente: Elaboración propia.

\section{CONCLUSIONES Y PROPUESTA DE MARCO COMPETENCIAL}

El presente artículo pretende definir qué significa el proceso de transformación digital de la administración pública y las implicaciones que éste tiene en relación con el perfil competencial del empleado público. Para ello se ha realizado un estudio de carácter mixto y exploratorio en dos fases: en primera lugar, una revisión narrativa e integradora de la literatura y, en segundo lugar, una investigación cualitativa basada en entrevistas a agentes claves vinculados con la transformación digital de la Administración Pública.

La transformación digital de la administración pública es un proceso de cambio generado por razones exógenas y endógenas y que tiene en la presencia y el uso de la tecnología su rasgo principal. Sin embargo, la tecnología, que es al mismo tiempo motor y meta del cambio, provoca una disrupción de carácter cultural que genera una revisión en profundidad de los artefactos, los procedimientos, los valores y las relaciones en la administración, y entre la administración, la ciudadanía y las empresas. Es decir, la transformación digital tiene su origen en la tecnología pero trasciende el uso de ésta para convertirse en una transformación cultural de la administración pública.

Como se afirma en el Plan de Digitalización de las Administraciones Públicas 2021-2025 (Gobierno de España, 2021a), «la Agenda España Digital 2025 recoge el impulso de la digitalización de la Administración Pública entre los diez ejes de reforma e inversión para catalizar una transformación digital que relance el crecimiento económico, la reducción de desigualdades, el aumento de la productividad y el aprovechamiento de todas las oportunidades que brindan las nuevas tecnologías». Así pues, la transformación digital de la Administración Pública es una palanca de transformación de todo el país, no sólo por el peso de la Administración Pública en el PIB sino por su influencia en la vida de ciudadanos y ciudadanas.

Por ello, la transformación digital de la Administración Pública se define como el proceso dinámico de mejora de la Administración pública a través de cambios culturales, organizativos y relacionales sustanciales para resolver problemas vinculados con la gobernanza, las personas, los servicios y los datos mediante una combinación de diferentes tecnologías: tecnologías de la información, de la computación, de la comunicación y de la conectividad.

Este proceso de transformación implica, de manera inevitable, cambios en la tarea y las cualidades del empleado público. Para atender a los cambios en el entorno, en la sociedad y en la propia administración, el empleado público desarrolla una serie de competencias, conocimientos y actitudes que le permiten prestar un buen servicio público en estos tiempos cambiantes. Es decir, la transformación digital se convierte en un camino de desarrollo (personal, ciudadano y profesional) del empleado público.

En este sentido, es urgente contar con un marco competencial que guíe la capacitación y el desarrollo del empleado público. Tener una imagen clara de cuáles son los perfiles competenciales del empleado público y cómo promoverlos es fundamental para luchar contra las debilidades y las amenazas señaladas 
por el Plan Nacional de Competencias Digitales (Gobierno de España, 2021b, p. 35) en su análisis DAFO de la Línea 5 («Formación en competencias digitales de las personas al servicio de las Administraciones Públicas»): desfase entre los procedimientos administrativos ya digitalizados y el uso eficiente de estos recursos telemáticos por parte del personal al servicio de las AA.PP; deficiencias organizativas que afectan a la cobertura de las necesidades funcionales no sólo para proporcionar capacitación en competencias digitales sino para poder usarlas de forma eficiente en el desarrollo de las tareas diarias del puesto de trabajo o riesgo elevado de que el déficit en capacitación de las personas al servicio de las AA.PP dificulte la adaptación al nuevo escenario donde las competencias digitales son esenciales para el desarrollo del trabajo diario.

Así pues, la nueva identidad del empleado público trasciende las competencias de la administración pública tradicional para incorporar una visión global de la persona que realiza una función pública. Esta visión se concreta, a la vista del análisis presentado en este artículo, en cinco competencias que representan cinco líneas de desarrollo integral para el empleado público (Figura 2):

FiguRA 2. COMPETENCIAS DEL EMPLEADO PÚBLICO EN UNA ADMINISTRACIÓN DIGITAL

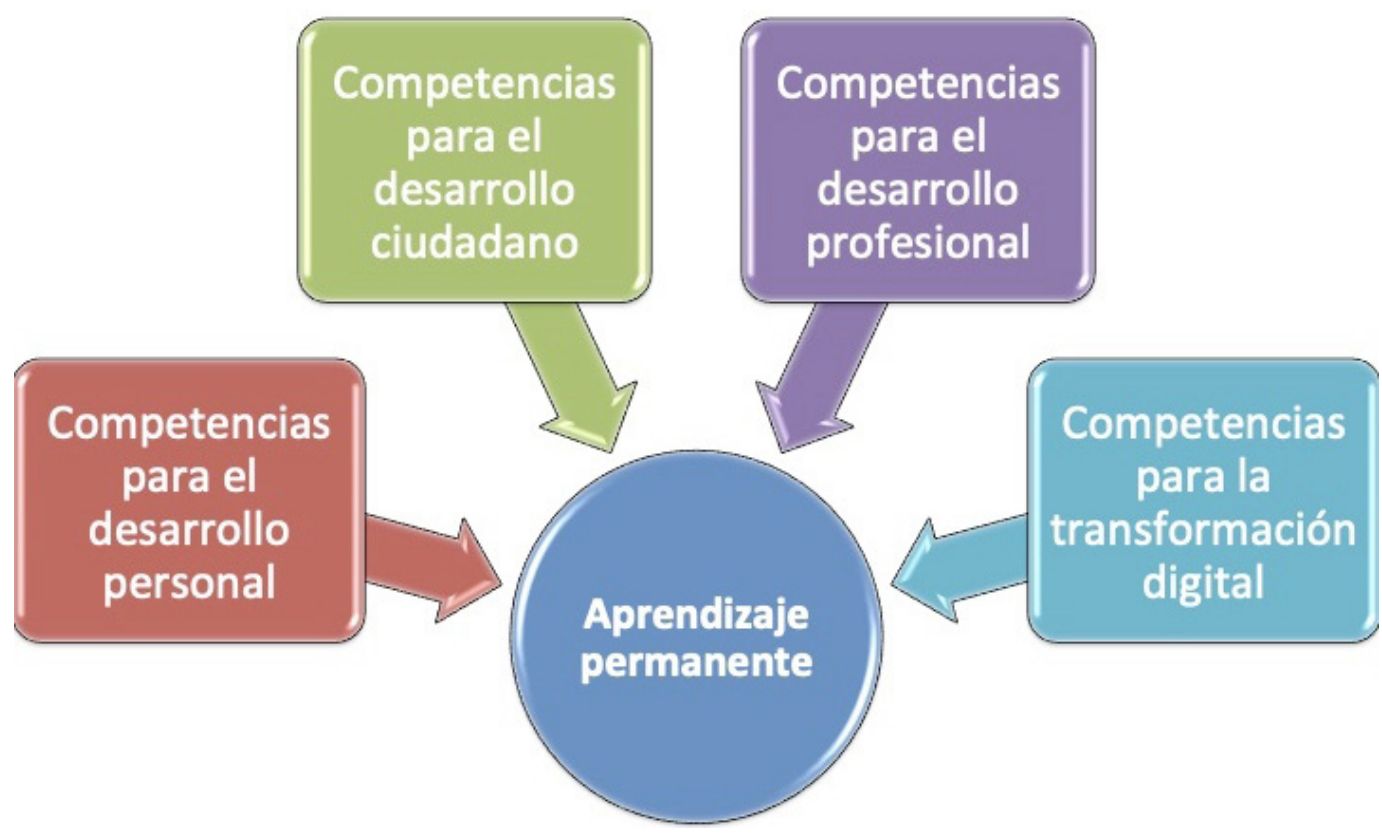

Fuente: Elaboración propia.

El desarrollo de estas cinco competencias, que depende tanto de procesos de aprendizaje formal como de aprendizajes informales, es flexible y se ajusta los distintos roles presentes en la administración pública, permitiendo así configurar perfiles de desempeño y de desarrollo precisos para cada unidad de la administración y cada puesto de trabajo.

Las cinco competencias se pueden articular a través de una serie de funciones que se ponen en funcionamiento en las distintas situaciones personales y profesionales en las cuales se encuentra el empleado público. De esta manera, las competencias se desarrollan mediante su uso además de a través de procesos de aprendizaje formal, representando un conocimiento dinámico y ajustado al entorno y a los problemas que surgen a lo largo de la vida personal y profesional (Figura 3).

En conclusión, sólo una Administración Pública y unos empleados y empleadas públicas competentes digitalmente podrán estar a la altura de los retos que la tecnología y la transformación digital plantean a toda la sociedad. Para ello, es necesario comprender qué significa transformación digital y cómo llevarla a la realidad de la administración pública a través de un marco de competencias digitales para el empleado público que contemple los elementos que han salido a la luz con esta investigación. Coincidimos, por tanto, con la conclusión de Buonocore et al. (2021): «las competencias digitales para la administración pública son esenciales para el proceso de modernización del país en todos los niveles, así que deberían ser una prioridad en el sistema de formación de los empleados públicos» (p. 88). 


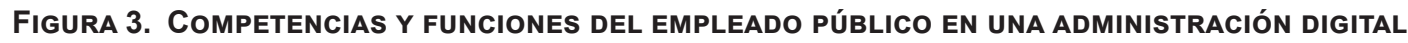

\begin{tabular}{|c|c|}
\hline $\begin{array}{l}\text { Aprendizaje } \\
\text { permanente }\end{array}$ & $\begin{array}{l}\text { - Gestión de la información } \\
\text { - Pensamiento crítico } \\
\text { - Pensamiento creativo e innovador } \\
\text { - Control del proceso de aprendizaje }\end{array}$ \\
\hline $\begin{array}{c}\text { Competencias para } \\
\text { el desarrollo } \\
\text { personal }\end{array}$ & $\begin{array}{l}\text { - Agencia } \\
\text { - Resiliencia } \\
\text { - Empatía } \\
\text { - Destrezas fisicas y ergonomía } \\
\text { - Destrezas socioemocionales } \\
\text { - Destrezas cognitivas y metacognitivas }\end{array}$ \\
\hline $\begin{array}{c}\text { Competencias para } \\
\text { el desarrollo } \\
\text { ciudadano }\end{array}$ & $\begin{array}{l}\text { - Conciencia global } \\
\text { - Compromiso con el entorno } \\
\text { - Compromiso con los derechos humanos y los ODS } \\
\text { - Cuidado de la salud y el medio ambiente } \\
\text { - Interacción con la diversidad }\end{array}$ \\
\hline $\begin{array}{c}\text { Competencias para } \\
\text { el desarrollo } \\
\text { profesional }\end{array}$ & $\begin{array}{l}\text { - Conocimiento del marco normativo } \\
\text { - Pertenencia y participación en la comunidad de práctica } \\
\text { - Liderazgo y colaboración } \\
\text { - Dominio de las alfabetizaciones múltiples aplicadas al puesto de } \\
\text { trabajo }\end{array}$ \\
\hline $\begin{array}{l}\text { Competencias para } \\
\text { la transformación } \\
\text { digital }\end{array}$ & $\begin{array}{l}\text { - Uso de dispositivos, software, plataformas y redes } \\
\text { - Alfabetización informacional y de datos } \\
\text { - Comunicación y colaboración en entornos digitales } \\
\text { - Creación de contenido digital } \\
\text { - Seguridad en entornos digitales } \\
\text { - Resolución de problemas }\end{array}$ \\
\hline
\end{tabular}

Fuente: Elaboración propia.

\section{REFERENCIAS BIBLIOGRÁFICAS}

Asgarkhani, M. (2005). Digital government and its effectiveness in public management reform: A local government perspective. Public Management Review, 7(3), 465-487. https://doi.org/10.1080/14719030500181227

Ballart, X. y Ramió, C. (2000). Ciencia de la Administración. Tirant lo Blanch.

Bankins, S., Denness, B., Kriz, A. y Molloy, C. (2017). Innovation agents in the public sector: Applying champion and promotor theory to explore innovation in the Australian public service. Australian Journal of Public Administration, 76(1), 122-137. http://dx.doi.org/10.1111/1467-8500.12198

Barcevičius, E., Cibaitè, G., Codagnone, C., Gineikytè, V., Klimavičiūtè, L., Liva, G., Matulevič, L., Misuraca, G., Vanini, I. y Misuraca, G. (2019). Exploring Digital Government transformation in the EU - Analysis of the state of the art and review of literature (EUR $29987 \mathrm{EN}$ ). Publications Office of the European Union. https://doi. org/10.2760/17207

Bouwman, R. y Grimmelikhuijsen, S. (2016). Experimental public administration from 1992 to 2014: A systematic literature review and ways forward. International Journal of PublicSector Management, 29(2), 110-131. http:// dx.doi.org/10.1108/IJPSM-07-2015-0129

Buonocore, F., Agrifoglio, R. y De Gennaro, D. (2021). The Role of Digital Competencies and Creativity for Job Crafting in Public Administration. En Metallo, C., Ferrara, M., Lazazzara, A., Za, S. (eds.), Digital Transformation and Human Behavior. Lecture Notes in Information Systems and Organisation, 37, 87-97. Springer, Cham. https://doi. org/10.1007/978-3-030-47539-0_7

Carretero, S., Vuorikari, R. y Punie, Y. (2017). DigComp 2.1: The Digital Competence Framework for Citizens with eight proficiency levels and examples of use (EUR 28558 EN). Publications Office of the European Union. https://doi. org/10.2760/38842

Casalino, N., Saso, T., Borin, B., Massella, E. y Lancioni, F. (2020). Digital Competences for Civil Servants and Digital Ecosystems for More Effective Working Processes in Public Organizations. En Agrifoglio, R., Lamboglia, R., Mancini, D. y Ricciardi, F. (eds.), Digital Business Transformation. Lecture Notes in Information Systems and Organisation, 38, 315-326. Springer, Cham. https://doi.org/10.1007/978-3-030-47355-6_21 
GAPP. Nueva Época - N. 27, noviembre 2021 - ISSN: 1989-8991 - DOI: https://doi.org/10.24965/gapp.i27.10923 - [Págs. 49-67]

Transformación digital de la administración pública: ¿Qué competencias necesitan los empleados públicos?

Fernando Trujillo Sáez / David Álvarez Jiménez

Castelnovo, W. y Sorrentino, M. (2018). The digital government imperative: a context-aware perspective. Public Management Review, 20(5), 709-725. https://doi.org/10.1080/14719037.2017.1305693

Clarke, A. (2020). Digital government units: what are they, and what do they mean for digital era public management renewal? International Public Management Journal, 23(3), 358-379. https://doi.org/10.1080/10967494.2019.1686447

Colbert, A., Yee, N. y George, G. (2016). The digital workforce and the workplace of the future. Academy of management journal, 59(3), 731-739. https://doi.org/10.5465/amj.2016.4003

Comisión Europea (CE) (2019). Key competences for lifelong learning (NC-02-19-150-EN-N). Publications Office of the European Union. https://dx.doi.org/10.2766/569540

Cortés Abad, O. (2020). La Administración tras el coronabreak. Políticas para ¿un nuevo paradigma administrativo? Gestión y Análisis de Políticas Públicas, 24, 6-23. https://doi.org/10.24965/gapp.i24.10811

Criado, J. I. y Gil-García, J. R. (2019). Creating public value through smart technologies and strategies: From digital services to artificial intelligence and beyond. International Journal of Public Sector Management, 32(5), 438-450. https://doi.org/10.1108/ijpsm-07-2019-0178

Döringer, S. (2020). "The problem-centred expert interview". Combining qualitative interviewing approaches for investigating implicit expert knowledge. International Journal of Social Research Methodology, 24(3), 265-278. https://doi.org/10.1080/13645579.2020.1766777

Frennert, S. (2019). Lost in digitalization? Municipality employment of welfare technologies. Disability and Rehabilitation: Assistive Technology, 14(6), 635-642. https://doi.org/10.1080/17483107.2018.1496362

Gil-García, J. R., Dawes, S. S. y Pardo, T. A. (2018). Digital government and public management research: finding the crossroads. Public Management Review, 20(5), 633-646. https://doi.org/10.1080/14719037.2017.1327181

Gobierno de España (2021a). Plan de Digitalización de las Administraciones Públicas (2021-2025). Ministerio de Asuntos Económicos y Transformación Digital. https://administracionelectronica.gob.es/pae_Home/pae_ Estrategias/Estrategia-TIC/Plan-Digitalizacion-AAPP.html

Gobierno de España (2021b). Plan Nacional de Competencias Digitales. Ministerio de Asuntos Económicos y Transformación Digital. https://portal.mineco.gob.es/RecursosNoticia/mineco/prensa/noticias/2021/210127_np_ digital.pdf

Haddaway, N. R., Collins, A. M., Coughlin, D. y Kirk, S. (2015). The role of Google Scholar in evidence reviews and its applicability to grey literature searching. PLOS ONE, 10(9). https://doi.org/10.1371/journal.pone.0138237

Kruyen, P. M. y Van Genugten, M. (2020). Opening up the black box of civil servants' competencies, Public Management Review, 22(1), 118-140. https://doi.org/10.1080/14719037.2019.1638442

Law, N., Woo, D., Torre, J. y Wong, G. (2018). A Global Framework of Reference on Digital Literacy Skills for Indicator 4.4.2. UNESCO Institute for Statistics. http://uis.unesco.org/sites/default/files/documents/ip51-global-frameworkreference-digital-literacy-skills-2018-en.pdf

Lee, J., Kim, B. J., Park, S., Park, S. y Oh, K. (2018). Proposing a value-based digital government model: Toward broadening sustainability and public participation. Sustainability, 10(9), 3078. https://doi.org/10.3390/su10093078

López-Fogués, A., Fernández-Baldor, A. y Boni, A. (2017). La innovación social digital colectiva y la administración. Propuestas para una visión más humana de la ciudad inteligente. Gestión y Análisis de Políticas Públicas, 18, 2342. https://doi.org/10.24965/gapp.v0i18.10427

Marangunić, N. y Granić, A. (2015). Technology acceptance model: a literature review from 1986 to 2013. Universal access in the information society, 14, 81-95. https://doi.org/10.1007/s10209-014-0348-1

Mergel, I., Edelmann, N. y Haug, N. (2019). Defining digital transformation: Results from expert interviews. Government Information Quarterly, 36(4), 101385. https://doi.org/10.1016/j.giq.2019.06.002

Munuera Gómez, M. P. (2016). Agenda digital: e-Servicios sociales. Gestión y Análisis de Políticas Públicas, 16, 27-46. https://doi.org/10.24965/gapp.v0i16.10362

OCDE (2017). Skills for a High Performing Civil Service. OECD Public Governance Reviews. OECDPublishing. http:// dx.doi.org/10.1787/9789264280724-en

Ouzzani, M., Hammady, H., Fedorowicz, Z. y Elmagarmid, A. (2016). Rayyan: a web and mobile app for systematic reviews. Systematic Reviews, 5(210). https://doi.org/10.1186/s13643-016-0384-4

Pereira, C. S., Durão, N., Fonseca, D., Ferreira, M. J. y Moreira, F. (2020). An Educational Approach for Present and Future of Digital Transformation in Portuguese Organizations. Applied Sciences, 10(3), 757. https://doi. org/10.3390/app10030757

Pittaway, J. J. y Montazemi, A. R. (2020). Know-how to lead digital transformation: The case of local governments. Government Information Quarterly, 37(4), 101474. https://doi.org/10.1016/j.giq.2020.101474

Romero Tarín, A. (2018). El paradigma de las Smart Cities en el marco de la gobernanza urbana. Gestión y Análisis de Políticas Públicas, 20, 29-35. https://doi.org/10.24965/gapp.v0i20.10536

Sala, A., Punie, Y., Garkov, V. y Cabrera Giraldez, M. (2020). LifeComp: The European Framework for Personal, Social and Learning to Learn Key Competence (EUR 30246 EN). Publications Office of the European Union. https://doi. org/10.2760/302967

Salvador Serna, M. y Ramírez Hernández, O. (2016). Gobierno abierto y competencias digitales: Transformando la administración pública para afrontar los retos del nuevo paradigma. Cuadernos de Gobierno y Administración Pública, 3(1), 1-13. https://doi.org/10.5209/CGAP.52992 
Schwab, K. (2016). La cuarta revolución industrial. Debate.

Snyder, H. (2019). Literature review as a research methodology: An overview and guidelines. Journal of Business Research, 104, 333-339. https://doi.org/10.1016/j.jbusres.2019.07.039

Starke, C., Baleis, J., Keller, B. y Marcinkowski, F. (2021). Fairness Perceptions of Algorithmic Decision-Making: A Systematic Review of the Empirical Literature. arXiv preprint. https://arxiv.org/abs/2103.12016

Vial, G. (2019). Understanding digital transformation: A review and a research agenda. The Journal of Strategic Information Systems, 28(2), 118-144. https://doi.org/10.1016/j.jsis.2019.01.003

World Economic Forum (WEF) (2015). New vision for education: Unlocking the potential of technology. British Columbia Teachers' Federation. http://www3.weforum.org/docs/WEFUSA_NewVisionforEducation_Report2015.pdf

Zhang, H., Xu, X. y Xiao, J. (2014). Diffusion of e-government: A literature review and directions for future directions. Government Information Quarterly, 31(4), 631-636. https://doi.org/10.1016/j.giq.2013.10.013

Zuiderwijk, A., Chen, Y.-C. y Salem, F. (2021). Implications of the use of artificial intelligence in public governance: A systematic literature review and a research agenda. Government Information Quarterly, 38(3), 101577. https://doi. org/10.1016/j.giq.2021.101577 